resuscitation before registering as general practitioners; handson courses on mannequins done at frequent intervals are also recommended. The National Committee on Confidential Enquiries into Maternal Deaths is promoting essential steps in the management of obstetric emergencies (ESMOE). This involves trainers visiting all health districts and carrying out training on mannequins and other visual aids on the emergency management of common conditions causing maternal deaths.

Previous SMRs have not identified cases of acute fatty liver of pregnancy (AFLP), but there is an impression that this condition does occasionally occur. ${ }^{2,3}$ Whether these deaths should be grouped under HDP is debatable. AFLP is defined as a condition that occurs abruptly in the second half of pregnancy and presents with increasing jaundice and right upper quadrant pain. The liver enzymes (transaminases) are abnormal and patients go on to develop coagulopathy and encephalopathy. Treatment involves supportive measures and delivery of the fetus. On pathological examination the liver shows fatty changes in the hepatocytes. ${ }^{12}$

There were 3 cases of maternal death grouped under AFLP. All took place at the same tertiary hospital and its geographical drainage area, all the patients were relatively young ( 26 - 30 years), parity 2 and in the second half of pregnancy, and all presented with jaundice and abdominal pain. One had mild hypertension, 2 had autopsies in keeping with fatty changes in the liver, and the third was said to have had all the clinical features of AFLP. As they were all from the same geographical area, the questions of viral infections, drug-related effects, or heavy alcohol intake are raised.

\section{Conclusion}

The majority of maternal deaths from hypertensive disorders in South Africa are associated with avoidable factors and substandard care. The challenge is to implement the recommendations arising from the SMRs. There is evidence from the 2005 - 2005 report that maternal deaths due to hypertension are decreasing, but more effort needs to be put into increasing the skills of health care professionals and improving the information on maternity care that we provide to women, fathers, families and communities.

1. National Committee on Confidential Enquiries into Maternal Deaths. Saving Mothers Fourth Repor 2005-2007. Pretoria: Department of Health, 2009.

2. National Committee on Confidential Enquiries into Maternal Deaths. Saving Mothers: Third Report on Confidential Enquiries into Maternal Deaths in South Africa 2002-2004. Pretoria: Department of Health, 2005 .

3. National Committee on Confidential Enquiries into Maternal Deaths. Saving Mothers: Third Report on Confidential Enquiries into Maternal Deaths in South Africa 1999-2001. Pretoria: Department of Health, 2006.

4. The Eclampsia Trial Collaborative Group. Which anticonvulsant for women with eclampsia? Evidence from the Collaborative Eclampsia Trial. Lancet 1995;345:1455-1463.

Langer A, Villar J, Tell K, et al. Reducing eclampsia related deaths - a call to action. Lancet 2008;371:705706.

6. Department of Health. Guidelines for Maternity Care in South Africa. 3rd ed. Pretoria; Department of Health, 2007

7. Department of Health. Essential Steps in the Management of Obstetric Emergencies. Pretoria Department of Health, 2008

Martin Jr NL, Thigpen MF Moore RC, et al. Stroke and pre-eclampsia and eclampsia: a paradigm shift focussing on systolic blood pressure. Obstet Gynecol 2005;105:246-254.

9. Neilson J. Pre-eclampsia and eclampsia. In: Lewis G and CEMACH. Why Mothers Die 2003-2005. London: RCOG Press, 2007: 72

10. Paruk F, Moodley J. Untoward effects of rapid-acting antihypertensive agents. In: Arukumaran S, Moodley J, eds. Best Practice and Research Clinical Obstetrics and Gynaecology. London: Harcour Publishers, 2001: 491-506.

11. Schutte J. Schuitmaker NW, van Roosmalen J, Steeger EA. Dutch Maternal Mortality Committee. Br Obstet Gynaecol 2008:115(6):732-736.

12. Barber MA, Equiluz I, Martin A, Placencia W, Valle L, Garcia JA. Acute fatty liver of pregnancy: analysis of five consecutive cases from a tertiary centre. J Obstet Gynecol 2010; 30(3):241-243.

\title{
CLINICAL IMAGES \\ Cytomegalovirus duodenitis, pseudotumour and cholangiopathy in advanced HIV
}

Bradley Yudelowitz, Adam Mahomed

Gastro-intestinal cytomegalovirus (CMV) infection manifests as oesophagitis, gastritis, enteritis and colitis. ${ }^{1,2}$ Reports of duodenal involvement are rare. ${ }^{3}$ We found only one reported CMV-induced pseudotumour of the duodenum and $14 \mathrm{CMV}$-induced gastrointestinal pseudotumours. ${ }^{4-6}$

Bradley Yudelowitz was a medical officer at the Reproductive Health and HIV Research Unit, University of the Witwatersrand, Johannesburg, at the time of writing.

Adam Mahomed is clinical head of the Division of Medical Gastroenterology, Department of Internal Medicine, Charlotte Maxeke Johannesburg Academic Hospital and University of the Witwatersrand.

\section{Case report}

A 35-year-old woman presented with cholestatic jaundice and partial bowel obstruction following 3 months' history of intermittent abdominal pain, nausea and vomiting. She had been diagnosed HIV positive 2 years before presentation, and had had no further followup. An abdominal ultrasound scan demonstrated splenic microabscesses, mesenteric and para-aortic lymphadenopathy and an indiscrete mass in the region of the head of the pancreas. A computed tomography (CT) scan (Fig. 1) confirmed a mass inseparable from the first part of the duodenum (D1) in the region of the head of the pancreas, compressing the common bile duct (CBD). Magnetic resonance cholangiopancreatography (MRCP) (Fig. 2) demonstrated dilated intrahepatic ducts and a long distal CBD stricture. Empiric tuberculosis (TB) treatment was started in view of the intraabdominal lymph nodes, splenic micro-abscesses and pancreatic mass, with poor clinical response.

Because of the patient's poor clinical response to TB treatment gastroscopy was performed, and an exophytic lesion at the second part of the duodenum (D2) was encountered which the scope was 


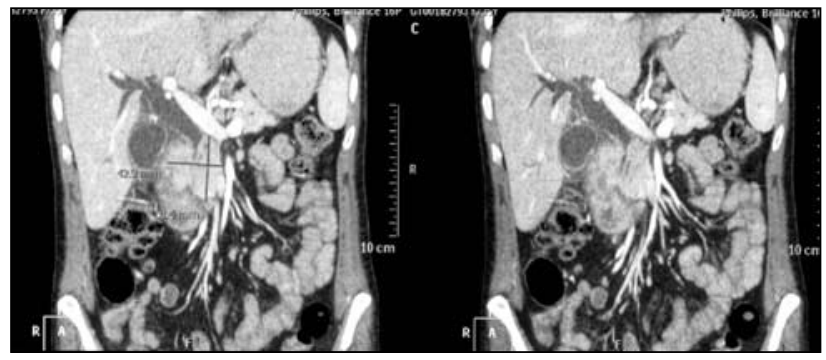

Fig. 1. CT scan-coronal view showing mass inseparable from duodenum.

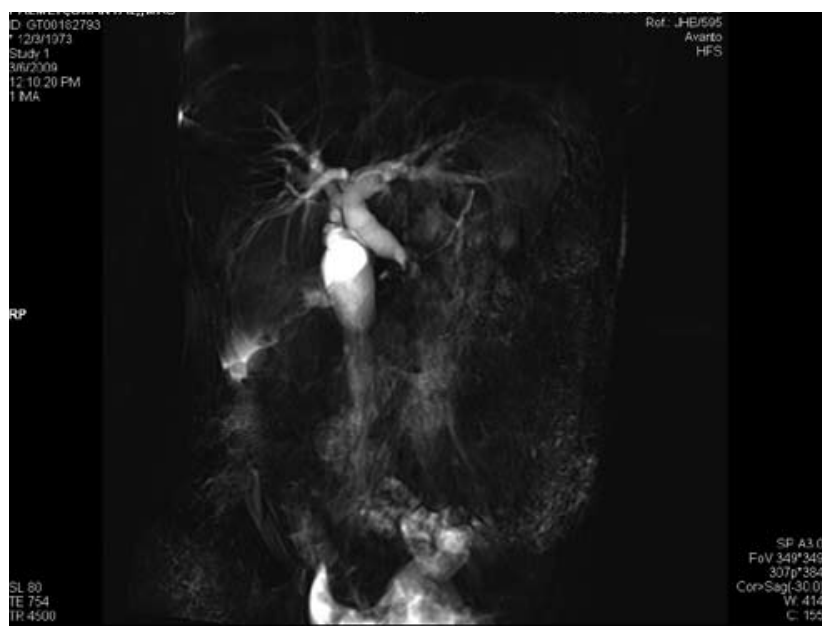

Fig. 2. MRCP demonstrating dilated intrahepatic biliary system.

unable to pass. Biopsies from this obstructing mass demonstrated a florid CMV duodenitis.

The patient was commenced on intravenous ganciclovir for 2 weeks, with a moderate clinical response but without complete resolution of inflammatory markers and liver function. Gastroscopy performed 1 week after completion of intravenous ganciclovir revealed almost complete resolution of the exophytic mass, and the endoscope was able to pass to the ampulla. Duodenal biopsy from the area of the mass demonstrated two poorly formed epitheloid granulomas within the lamina propria, with a Ziehl-Neelsen stain highlighting several acid-fast bacilli. No viral cytopathic effect was noted in this specimen. At this stage she had been on TB treatment for 1 month and had been treated with ganciclovir for 2 weeks.

Endoscopic retrograde cholangiopancreatography was done because liver function did not improve and the patient experienced ongoing right upper quadrant pain. The duodenal cap was markedly diseased, and mildly dilated intrahepatic ducts were demonstrated with no distal filling of the CBD in keeping with a long distal CBD stricture. It was considered that the stricture was not typical of extrinsic compression, and a wide sphincterotomy and stent was inserted. To confirm CMV as the pathogen responsible for the CBD stricture, biopsy of the $\mathrm{CBD}$ and/or culture of the bile aspirate for CMV should ideally have been undertaken. The patient was discharged on oral ganciclovir for 3 weeks and continuing standard $\mathrm{TB}$ treatment. Antiretrovirals were initiated within a few weeks of discharge, comprising lamivudine, stavudine and efavirenz, at a CD4 count of 53 cells $/ \mu l$.

The patient improved slowly over the next few months. She continued taking antiretrovirals with minimal complications, and completed 9 months of TB treatment. At the time of writing she was clinically well with an undetectable viral load and reported improvements in all aspects of her life including work and leisure.
She reported a return of libido and was practising safe sex with a discordant partner. A repeat endoscopy one year after her initial presentation demonstrated a normal duodenum with unremarkable histology. Liver function improved significantly and was stable at the time of writing.

\section{Discussion}

$\mathrm{CMV}$ is an important pathogen, causing a wide spectrum of disease from asymptomatic infection to mononucleosis syndrome in immunocompetent individuals to disseminated disease in immunocompromised individuals.

Once acquired by symptomatic or asymptomatic primary infection, CMV persists indefinitely in various cell types and organs including salivary glands, lung, liver, kidney, bowel, pancreas, adrenal glands and central nervous system. The cellular inflammatory response consists of plasma cells, lymphocytes and monocyte-macrophages. Granulomatous reactions occasionally develop, usually in the liver. Infection will usually remain latent. Reactivation syndromes frequently develop when T-lymphocyte-mediated immunity is compromised.

Active significant CMV infection is sometimes difficult to diagnose definitively in patients with advanced HIV disease because of its propensity to infect rapidly dividing cells and the frequent presence of other opportunistic infections. ${ }^{1}$ Fatal CMV infection is often associated with persistent viraemia and the involvement of multiple organ systems, often in conjunction with another opportunistic infection such as TB. The guidelines for ganciclovir use remain anecdotal.

CMV is often noted in pathological specimens and should not be viewed as incidental, even in the presence of another infection. CMV pseudotumour should be considered as a differential diagnosis in mass lesions of the gastro-intestinal tract in the immunocompromised patient. The tumour does respond to antiviral treatment. ${ }^{4}$

The exact incidence and cause of HIV cholangiopathy has yet to be determined, but infection-related damage to the biliary tree has been suggested and CMV infection appears to be one possible cause.

This is the first reported case of CMV-related duodenitis, pseudotumour and cholangiopathy of which we are aware. CMV is emerging as an important pathogen in patients with advanced HIV and other immunocompromised hosts. We propose that CMV infection associated with a concurrent infection is clinically significant in such patients. It should not be overlooked even in the presence of other opportunistic infection, and further research is required into how best to treat CMV and HIV co-infection.

Approval was obtained from the Ethics Committee of the University of the Witwatersrand. There were no conflicts of interest. Written consent was obtained from the patient.

\footnotetext{
Mong A, Levine MS, Furth EE, Laufer I. Cytomegalovirus duodenitis in an AIDS patient. AJR Am J Roentgenol 1999;172(4):939-940.

2. Goodgame RW. Gastrointestinal cytomegalovirus disease. Ann Intern Med 1993;119(9):924-935.

3. Wilcox CM, Schwartz DA. Symptomatic CMV duodenitis. An important clinical problem in AIDS. J Clin Gastroenterol 1992;14(4):293-297.

4. Kelesidis T, Tozzi S, Mitty R, Worthington M, Fleisher J. Cytomegalovirus pseudotumor of the duodenum in a patient with AIDS: an unrecognized and potentially treatable clinical entity. Int $J$ Infect Dis 2010;14(4):e274-282.

5. Mohan H, Bal A, Garg S, Dalal U. Cytomegalovirus-associated pseudotumor simulating gastric malignancy in acquired immunodeficiency syndrome: a case report with review of literature. Jpn J Infect Dis 2007;60(2-3):134-136. Review.

6. Wisser J, Zingman B, Wasik M, Duva-Frissora A, Beazley R, McAneny D. Cytomegalovirus pseudotumor presenting as bowel obstruction in a patient with acquired immunodeficiency syndrome. Am J Gastroenterol 1992;87(6):771-774.
} 